\title{
Effect of the electron density stratification on off-limb $O$ VI line profiles: How large is the velocity distribution anisotropy in the solar corona?
}

\author{
N.-E. Raouafi ${ }^{\star}$ and S. K. Solanki
}

\author{
Max-Planck-Institut for Solar System Research ${ }^{\star \star}$, Max-Planck-Strasse 2, 37191 Katlenburg-Lindau, Germany \\ e-mail: [raouafi; solanki]@linmpi.mpg.de
}

Received 29 December 2003 / Accepted 21 July 2004

\begin{abstract}
Coronagraphic spectral observations carried out with UVCS on SoHO have shown that the velocity distribution in the solar corona is highly anisotropic. Here we examine the influence of the density stratification on the interpretation of such observations. In particular, we investigate the profiles of O VI lines emitted in the corona by employing an analytical 2-D model of the large scale coronal magnetic field and solar wind. We concentrate on the polar coronal holes and take into account the integration along the line of sight. We find that at distances greater than $1 R_{\odot}$ from the solar surface the widths of the emitted lines are significantly affected by the details of the adopted electron density profiles. In particular, the densities deduced by Doyle et al. (1999a,b) from SoHO data result in O VI profiles whose widths and intensity ratio are relatively close to the values observed by UVCS/SoHO although only isotropic velocity distributions are employed. Hence we expect that the magnitude of anisotropy of the velocity distribution deduced from UVCS data depends strongly on the adopted density profile.
\end{abstract}

Key words. line: profiles - scattering - Sun: corona - Sun: magnetic fields - Sun: solar wind - Sun: UV radiation

\section{Introduction}

Extreme-ultraviolet (EUV) spectral lines provide valuable information on the plasma conditions in the solar wind acceleration zone. Information on the densities, the temperatures, the bulk velocities and the velocity distributions of different species can be obtained from the total intensity, Doppler width and shift of multiple line profiles with the appropriate instrument, such as the UltraViolet Coronagraph Spectrometer (UVCS; Kohl et al. 1995, 1997) onboard the Solar and Heliospheric Observatory (SoHO; Domingo et al. 1995).

One of the most exciting results obtained from data recorded by UVCS is the large width of spectral lines emitted by heavy ions (namely O VI and $\mathrm{Mg} \mathrm{X}$ ) in polar coronal holes during the minimum of solar activity. Cranmer et al. (1999c) carried out a thorough analysis and detailed interpretation of the data. In particular, they took into account the integration along the line of sight and the superradial expansion of the magnetic field consistent with the white light images (for details see Cranmer et al. 1999c). In order to reproduce simultaneously the large observed line widths and values near or below unity of the intensity ratio between the $\mathrm{O}$ VI lines at $\lambda 1031.92$ and $\lambda 1037.61$, a significant anisotropy in the velocity distributions of the heavy ions was invoked, corresponding to a kinetic

* Associated researcher to the LERMA Departement at the Observatoire de Paris-Meudon, France.

$\star \star$ Previously known as Max-Planck-Institut für Aeronomie. temperature anisotropy: $10<T_{\perp} / T_{\|}<100$ (see Kohl et al. 1997; Li et al. 1998; Cranmer et al. 1999a,b,c and others). In addition, the heavy ions are deduced to be hotter and faster than the protons. This result has been interpreted in terms of ioncyclotron waves propagating through the corona (Kohl et al. 1999; Cranmer et al. 1999a,b,c; and others), which has an impact on the mechanisms of acceleration and heating of the particles of the solar wind.

A number of complications face the interpretation of the observations of the solar corona, not the least of which is introduced by the low opacity of coronal gas. This implies, among other things, that the observer collects photons emitted over a large range of distances along the LOS, so that the measured signal is due to coronal plasma under a variety of physical conditions (densities, temperatures, absolute and line of sight (LOS) velocities, magnetic field, etc.). In general, integrating along the line of sight (LOS) enhances the line width due to contributions from the LOS component of the solar-wind velocity, particularly in the presence of the superradial expansion of the Sun's open magnetic field (Cranmer et al. 1999a,b,c). The electron density profile plays a key role in determining the intensities and shapes of LOS integrated profiles, since the solar outflow speed and consequently the LOS speed strongly depend on the variation of the electron density with heliocentric distance.

In the present paper we consider the influence of the exact choice of the adopted density stratification on the LOS 


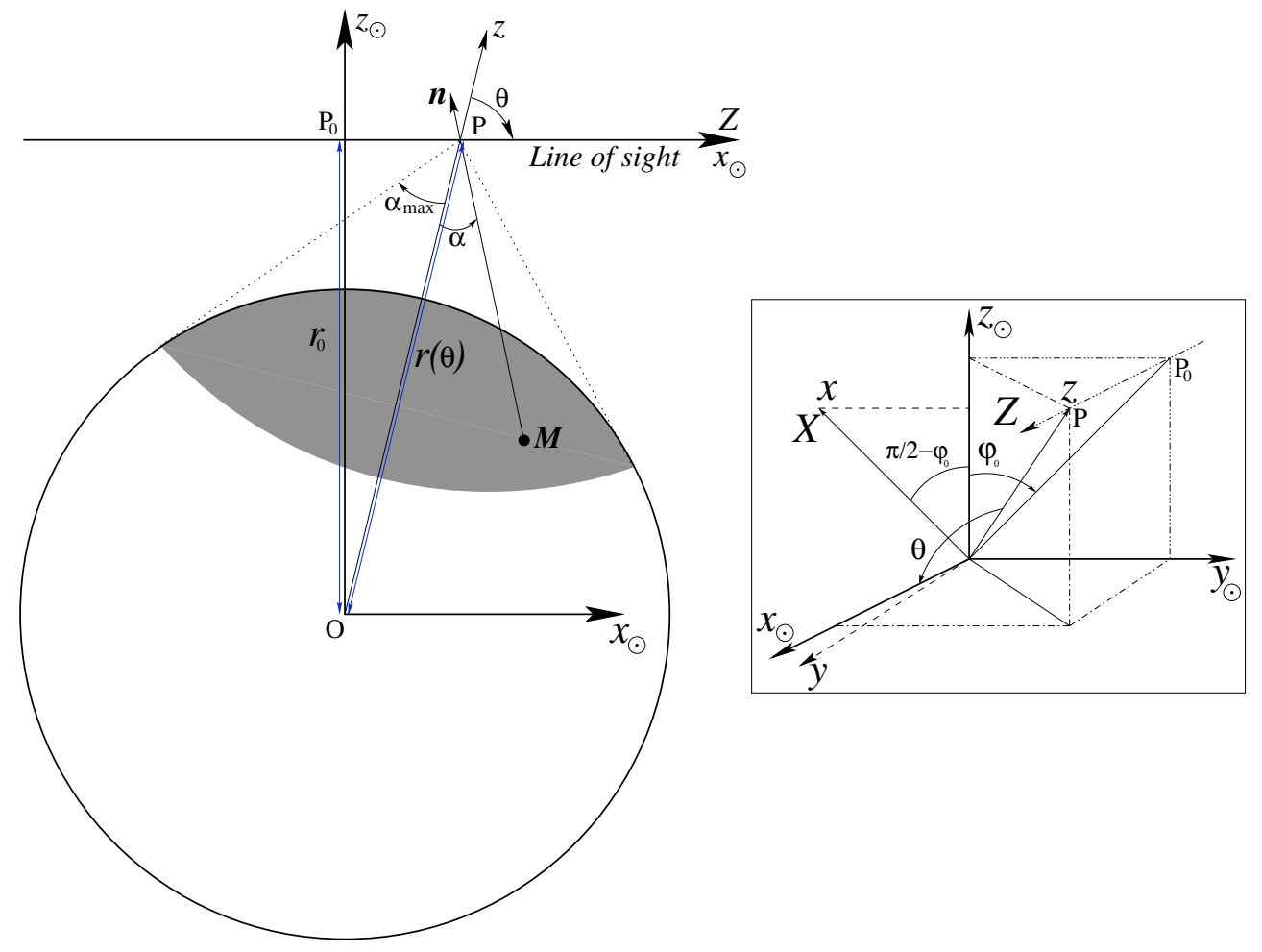

Fig. 1. Definition of the different axes and angles used for the calculation of the spectral profiles of coronal lines emitted in the direction of the line of sight $(P Z)$ which is parallel to $\left(O x_{\odot}\right)$ (see right figure). The axis $(P x)$ is orthogonal to the plane of the left figure and lies in the plane of the right one, while the directions of $(P y)$ and $(P z)$ change in the scattering plane $\left(O P_{0} Z\right)$ to form an orthogonal coordinate system. For each point on the line of sight the emitting atoms or ions interact with electrons with an isotropic velocity distribution and with the radiation coming from the transition region located in the spherical cap (shaded area on the solar disk). The unit vector $\boldsymbol{n}$ characterizes the direction of propagation of radiation coming from a small area around $M$. The angular coordinates of $\boldsymbol{n}$ are $\alpha$ (polar angle $\equiv(\widehat{\boldsymbol{n}, p} z)$ ) and the azimuth angle $\beta$ (not plotted) that is the angle between $(P x)$ and the projection of $\boldsymbol{n}$ on the plane $(P x y)$. The angle $\varphi_{0}=\left(O z_{\odot}, O P_{0}\right)$ together with $r_{0}$ define the line of sight.

integrated profiles of the O VI doublet in a polar coronal hole. We employ a simple, but successful model of the large-scale magnetic field at solar minimum and a consistent treatment of the solar wind.

\section{Geometry of the system: Different reference frames}

Three different reference frames are used in the present model. The choice of the axes in the different frames is arbitrary and can be changed. The reference frames used here are shown in Fig. 1 and are

* The heliocentric frame $\left(O x_{\odot} y_{\odot} z_{\odot}\right)$ : The employed magnetic field given by Banaszkiewicz et al. (1998) is defined in this frame. $O$ is the center of the Sun; the axis $\left(O x_{\odot}\right)$ is parallel to the line of sight; the axis $\left(O y_{\odot}\right)$ lies in the equator and is oriented from east to west in the plane of the sky. Finally, $\left(O z_{\odot}\right)$ is the polar axis which is assumed to be in the plane of the sky, oriented from the south to the north pole. $\left(O y_{\odot} z_{\odot}\right)$ spans the plane of the sky.

* The local solar frame $(P x y z)$ : This frame is useful for computing the scattering process of the radiation coming from the solar disk. $P$ is a given point on the line of sight at which we consider the line formation, $(P z)$ is the axis joining $O$ and $P ;(P x)$ is the axis perpendicular to $(P z)$ and lies in the plane of the sky $((P x)$ is parallel to the limb and perpendicular to the LOS). The orientation of $(P x)$ is such that $(P y)$ is always in front of the plane of the sky for a right handed system of axes. The two axes $(P y)$ and $(P z)$ together form the scattering plane which contains the line of sight. The scattering angle $\theta$ is the angle between $(P z)$ and the line of sight $(P Z)$ or $\left(O x_{\odot}\right)$. The projection of $(P z)$ on the plane of the sky $\left(P y_{\odot} z_{\odot}\right)$ makes an angle $\varphi_{0}$ with the polar axis $\left(P z_{\odot}\right)$. This angle together with $r_{0}\left(=O P_{0}\right)$ uniquely identifies the line of sight.

* The line of sight frame $(P X Y Z):(P X) \equiv(P x) ;(P Z) \equiv$ $\left(O x_{\odot}\right)$ is the LOS axis and $(P Y)$ is parallel to the projection of $(P z)$ on the plane of the sky but with opposite orientation. This system is the most convenient one for the integration along the line of sight.

$r(\theta)(=O P)$ is the distance of point $P$ on the LOS to the center of the Sun, while $r_{0}$ represents the distance of $P_{0}$ from the center of the Sun. The quantities $r_{0}\left(=O P_{0}\right), r(\theta)$ and $r_{0}$ are related by

$r(\theta)=-\frac{r_{0}}{\sin \theta}$.

For a given point $P$ on the line of sight the scattering atoms (or ions) are illuminated by the radiation coming from the chromosphere-corona transition region in a spherical cap 


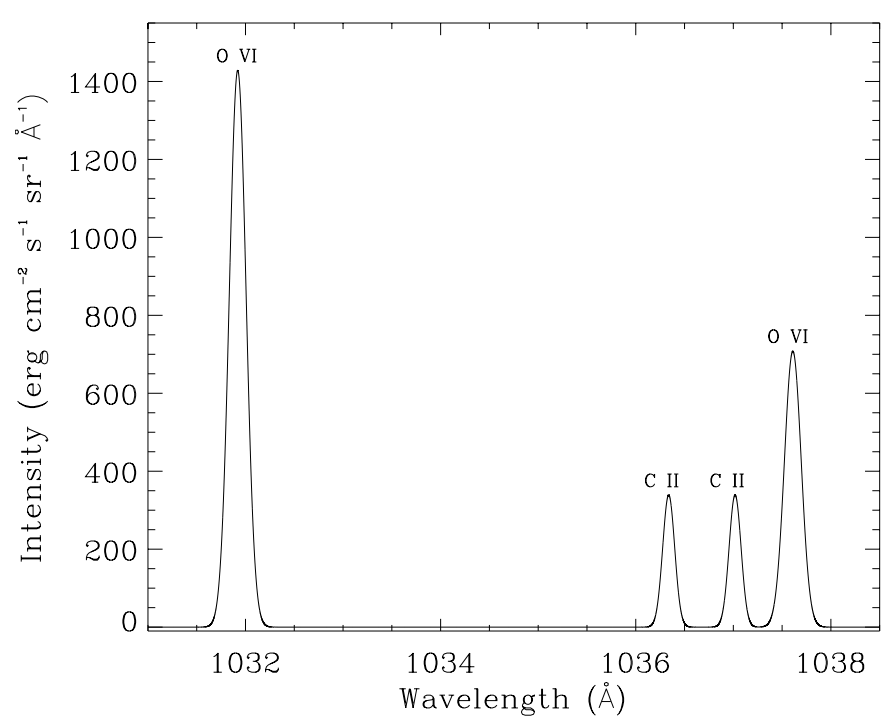

Fig. 2. The incident spectrum used in the calculation of the LOS integrated profiles of the O VI doublet. The radiance of O VI $\lambda 1031.92$

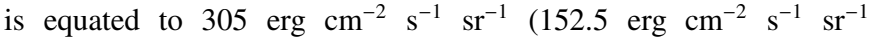
for O VI 11037.61). For each member of the $\mathrm{C}$ II doublet the radiance is $52 \mathrm{erg} \mathrm{cm}^{-2} \mathrm{~s}^{-1} \mathrm{sr}^{-1}$. We assume also the same widths for the $\mathrm{O}$ vi doublet $\left(35 \mathrm{~km} \mathrm{~s}^{-1}\right)$ and for the $\mathrm{C}$ II lines $\left(25 \mathrm{~km} \mathrm{~s}^{-1}\right)$.

(bounded by the dotted lines in Fig. 1). Any radiation beam coming from an infinitesimal area around the point $M$ is characterized by the unitary vector $\boldsymbol{n}$ that is defined by the polar angle $\alpha=(\widehat{O z, n})$ and the azimuth angle $\beta$ between $(P x)$ and the projection of $\boldsymbol{n}$ on the plane $(P x y)$. The area of this spherical cap depends on the location of the scattering atom on the line of sight. We can characterize this area by the angle $\alpha_{\max }$, which is given as a function of the scattering angle $\theta$ by

$\alpha_{\max }(\theta)=\arctan \left(\frac{R_{\odot}}{\sqrt{r(\theta)^{2}-R_{\odot}^{2}}}\right)$.

\section{Line formation}

\subsection{Computed spectral lines}

We compute the intensity profiles of the lines of the O VI doublet ( $\lambda 1031.92$ and $\lambda 1037.61)$. The O VI ion emits a doublet at $\lambda 1031.92 \AA$ and $\lambda 1037.61 \AA$ that corresponds to the atomic transitions $1 \mathrm{~s}^{2}{ }^{1} \mathrm{~S} \quad 2 \mathrm{~s} \quad{ }^{2} \mathrm{~S}_{1 / 2}-1 \mathrm{~s}^{2} \quad{ }^{1} \mathrm{~S} \quad 2 \mathrm{p} \quad{ }^{2} \mathrm{P}_{3 / 2}$ and $1 s^{2}{ }^{1} \mathrm{~S} 2 \mathrm{~s}{ }^{2} \mathrm{~S}_{1 / 2}-1 \mathrm{~s}^{2}{ }^{1} \mathrm{~S} 2 \mathrm{p}{ }^{2} \mathrm{P}_{1 / 2}$, respectively. In the solar corona, these lines are formed by electron collisions and the resonance scattering of the radiation coming from the solar disk. We take into account the effect of the solar wind (Doppler dimming) and also the optical pumping of the OVI $\lambda 1037.61$ line by the chromospheric C II doublet $(\lambda 1036.3367, \lambda 1037.0182)$. This process is important above $\approx 2 R_{\odot}$ where the solar wind speed reaches values that enable optical pumping to occur. The incident solar disk spectrum considered for the calculations is shown in Fig. 2. All the line profiles are assumed to be Gaussians. In the present computations, the radiance of the $\mathrm{O}$ VI doublet $\lambda 1031.92$ and $\lambda 1037.61$ are equated to 305 and $152.5 \mathrm{erg} \mathrm{cm}^{-2} \mathrm{~s}^{-1} \mathrm{sr}^{-1}$, respectively. We assume also the same profiles for the $\mathrm{C}$ II doublet with widths of $25 \mathrm{~km} \mathrm{~s}^{-1}$ and radiances of $52 \mathrm{erg} \mathrm{cm}^{-2} \mathrm{~s}^{-1} \mathrm{sr}^{-1}$ each. We use the limb-brightening measured by Raouafi et al. (2002) for the $\mathrm{O}$ VI lines and no limb-brightening for the $\mathrm{C}$ II doublet (see Warren et al. 1998). For comparison, different values adopted by different authors are presented in Table 1 .

\subsection{Theory of line formation in the corona}

Raouafi (2000, 2002) presented the general theory describing the formation of the Stokes parameters of a resonance scattering spectral line sensitive to the Doppler redistribution and Hanle effects. He gives the Stokes parameters as a function of the velocity vector of the scattering atoms (or ions) and the local magnetic field vector (the coronal magnetic field vector in the solar case) in which they are immersed.

The frequency-dependent intensity of a given spectral line is obtained through the equation

$$
\mathcal{I}(v, r)=\int_{-Z_{0}}^{+Z_{0}}\left[\mathcal{I}_{\text {col }}(v, r, Z)+\mathcal{I}_{\text {rad }}(v, r, Z)\right] \mathrm{d} Z,
$$

where $\mathcal{I}_{\text {col }}(v, r, Z)$ and $\mathcal{I}_{\text {rad }}(v, r, Z)$ are the collisionally and radiatively excited components, respectively, $v$ is the frequency, $r$ is the heliocentric distance and $Z$ is the coordinate along the LOS. $\mathcal{I}_{\text {col }}(v, r, Z)$ is a function of local quantities only, while $\mathcal{I}_{\text {rad }}(v, r, Z)$ also depends on the incident line profile. In the case of the considered $\mathrm{O}$ VI lines these are the disk emission profiles arising from the transition region. Both the electronic and radiative contributions depend on the velocity distribution (microscopic and macroscopic atomic motions) of the scattering ions through Doppler redistribution.

The contributions to $\mathcal{I}_{\text {col }}(v, r, Z)$ and $\mathcal{I}_{\text {rad }}(v, r, Z)$ of a given point on the line of sight characterized by a scattering angle $\theta$ are, respectively, given by

$$
\begin{aligned}
\mathcal{I}_{\text {col }}(v, \theta)= & h v_{l u} \frac{N_{\mathrm{i}}(\theta) N_{\mathrm{e}}(\theta) \alpha_{l u}\left(T_{\mathrm{e}}\right)}{4 \pi} F(v, \theta), \\
\mathcal{I}_{\text {rad }}(v, \theta)= & h v_{l u} \frac{N_{\mathrm{i}}(\theta) B_{l u}}{(4 \pi)^{2}} \int_{\Omega} \mathrm{d} \Omega \sin \alpha F(\boldsymbol{v}, \theta) \\
& \times \mathcal{I}_{\text {inc }}(v, \Omega, \theta) \mathcal{M}(\Omega, \theta) .
\end{aligned}
$$

Here, $N_{\mathrm{i}}(\theta)$ is the number of scattering ions per unit volume, $N_{\mathrm{e}}(\theta)$ is the electron density $\left(\mathrm{cm}^{-3}\right)$ and $T_{\mathrm{e}}(\theta)$ is the electron temperature. $\alpha_{l u}\left(T_{\mathrm{e}}\right)$ is the coefficient of electronic collisions and $B_{l u}$ is the Einstein coefficient for absorption. $F(v, \theta)$ is the velocity field distribution of the scattering ions. In the following we limit ourselves to the effect of the Doppler redistribution alone (i.e. we consider the zero magnetic field limit for line formation, although the influence of the magnetic field on the solar wind is taken into account in a simple manner (Sect. 4)). $\mathcal{I}_{\text {inc }}(\nu, \Omega, \theta)$ is the intensity profile of the incident line as a function of the frequency $v$ and the direction of incidence given by the angles $(\alpha, \beta)$. $\mathcal{M}(\Omega, \theta)$ is the phase matrix. In the case where only the Doppler redistribution is taken into account, it is described by (see Sahal-Bréchot et al. 1998; Raouafi 2000, 2002)

$$
\begin{aligned}
\mathcal{M}(\Omega, \theta)= & 1+\frac{\mathcal{W}_{u l}}{8}\left[\left(3 \cos ^{2} \theta-1\right)\left(3 \cos ^{2} \alpha-1\right)\right. \\
& \left.-3\left(\sin 2 \theta \sin 2 \alpha \sin \beta+\sin ^{2} \theta \sin ^{2} \alpha \cos 2 \beta\right)\right] .
\end{aligned}
$$


Table 1. Solar disk spectral parameters of the O VI and C II doublets obtained by different authors. The widths are those given for the center of the solar disk. Erdélyi et al. (1998) found that the line widths change from center to limb by approximately $20 \%$ for O VI $\lambda 1037.61$ and by $40 \%$ for $\mathrm{C}$ II $\lambda$ 1036.3367. These variations are not taken into account in our calculations.

\begin{tabular}{|c|c|c|c|c|c|}
\hline Parameter & Reference & O vi $\lambda 1031.92$ & C II $\lambda 1036.3367$ & C II $\lambda 1037.0182$ & O VI $\lambda 1037.61$ \\
\hline \multirow{4}{*}{$\begin{array}{l}\text { Radiance } \\
\left(\mathrm{erg} \mathrm{cm}^{-2} \mathrm{~s}^{-1} \mathrm{sr}^{-1}\right)\end{array}$} & Noci et al. (1987) & 305 & - & 52 & 152 \\
\hline & Warren et al. (1998) & 341-481 (Av. 402) & 52 & - & - \\
\hline & Wilhelm et al. (1998) & 248 & 35.9 & 44.8 & 118 \\
\hline & Gabriel et al. (2003) & 333.5 & 49.7 & 57.7 & 159.5 \\
\hline \multirow{4}{*}{$\begin{array}{l}\text { Width }\left(\mathrm{e}^{-1}\right) \\
\left(\mathrm{km} \mathrm{s}^{-1}\right)\end{array}$} & Noci et al. (1987) & $29.3(101 \mathrm{~mA})$ & - & $20.8(72 \mathrm{m \AA})$ & 29.3 \\
\hline & Warren et al. (1997) & 40 (136 mÅ) & $25^{*}$ & $25^{*}$ & $37(127 \mathrm{m \AA})$ \\
\hline & Erdélyi et al. (1998) & - & $25(85 \mathrm{~m} \AA)$ & - & $36(126 \mathrm{~mA})$ \\
\hline & Gabriel et al. (2003) & 29.3 & 20.8 & 20.8 & 29.3 \\
\hline
\end{tabular}

* Non-thermal widths.

The expression for $\mathcal{W}_{u l}$ is given by Landi Degl'Innocenti (1984). According to it we have for the two O VI resonance lines

$W_{u l}=\left\{\begin{array}{c}0 \text { for }\left(J_{u}, J_{l}\right)=\left(\frac{1}{2}, \frac{1}{2}\right), \\ 0.5 \text { for }\left(J_{u}, J_{l}\right)=\left(\frac{3}{2}, \frac{1}{2}\right) .\end{array}\right.$

The intensity profile as a function of the scattered radiation frequency $v$ is obtained after a double integration over the velocity field components $v_{X}$ and $v_{Y}$ in the plane perpendicular to the line of sight:

$\mathcal{I}(v) \mathrm{d} v=\mathrm{d} v_{Z} \int \mathrm{d} \theta \iint \mathcal{I}(\boldsymbol{v}) \mathrm{d} v_{X} \mathrm{~d} v_{Y}$.

The expression given by Eq. (7) is general. In particular, no assumption is made regarding the incident line profile or the velocity distribution of the emitting atoms or ions.

We assume a Maxwellian velocity distribution with a drift velocity vector $\boldsymbol{u}$ for the scattered ions inside the coronal hole that is given by

$$
F(\boldsymbol{v})=\frac{1}{\pi^{3 / 2} \alpha_{s}^{3}} \exp \left[-\left(\frac{\boldsymbol{v}-\boldsymbol{u}}{\alpha_{s}}\right)^{2}\right],
$$

where $v$ is the atoms' microscopic velocity vector, $\alpha_{s}$ is the width of the microscopic distribution and $\boldsymbol{u}$ is the macroscopic outflow velocity vector of the scattering atoms (ions) that can be equated to the solar wind outflow velocity vector. Note that no anisotropy in the kinetic temperature of the scattering ions is prescribed.

We further assume that the incident light has a Gaussian profile that is given by

$$
\mathcal{I}_{\text {inc }}(\nu, \Omega, \theta)=\frac{\mathcal{I}_{\mathrm{R}} f(\Omega, \theta)}{\sqrt{\pi} \alpha_{i}} \exp \left[-\left(\frac{\boldsymbol{v} \cdot \boldsymbol{n}}{\alpha_{i}}\right)^{2}\right],
$$

where $\mathcal{I}_{\mathrm{R}}$ is the radiance of the line emitted by the solar disk (in erg $\left.\mathrm{cm}^{-2} \mathrm{~s}^{-1} \mathrm{sr}^{-1}\right), \boldsymbol{v} \cdot \boldsymbol{n}=\frac{c}{v_{0}}\left(v-v_{0}\right), \alpha_{i}$ is the Doppler width of the incident profile and $f(\Omega, \theta)$ is a function describing the variations of the emission on the disk (limb-brightening or limb-darkening, active regions, etc.). Above the poles, the region of interest in this paper, solar rotation affects line profiles only in second order, so that it does not need to be explicitely included in Eq. (9). Also, the magnitude of the solar rotation speed is small compared with the widths of the incident profile or of the turbulence velocity distribution, which further reduces its influence.

After integration over the velocity components $v_{X}$ and $v_{Y}$, we obtain for the component excited by electron collisions

$$
\begin{aligned}
\mathcal{I}_{\mathrm{col}}(\nu, \theta)= & h v_{l u} \frac{N_{\mathrm{i}}(\theta) N_{\mathrm{e}}(\theta) \alpha_{l u}\left(T_{\mathrm{e}}\right)}{4 \pi} \\
& \times \frac{1}{\sqrt{\pi} \alpha_{s}} \exp \left[-\left(\frac{v_{Z}-u_{Z}}{\alpha_{s}}\right)^{2}\right],
\end{aligned}
$$

where $u_{Z}$ is the component along the line of sight of the scattering ion velocity. For the component excited by radiation we obtain

$$
\begin{aligned}
\mathcal{I}_{\mathrm{rad}}(\nu, \theta)= & h v_{l u} \frac{N_{\mathrm{i}}(\theta) B_{l u} \mathcal{I}_{\mathrm{R}}}{(4 \pi)^{2}} \int_{\Omega(\theta)} \mathrm{d} \Omega \sin \alpha f(\Omega, \theta) \\
& \times \mathcal{D}(\Omega, \theta) \mathcal{P}(\Omega, \theta) \mathcal{M}(\Omega, \theta),
\end{aligned}
$$

where $\mathcal{D}(\Omega, \theta)$ and $\mathcal{P}(\Omega, \theta)$ are, respectively, the terms giving the Doppler dimming and the radiative profile. They are given by

$$
\begin{aligned}
\mathcal{D}(\Omega, \theta)= & \frac{\exp \left[-\left(\frac{\boldsymbol{u} \cdot \boldsymbol{n}}{\left[\alpha_{i}^{2}+\alpha_{s}^{2}\right]^{1 / 2}}\right)^{2}\right]}{\left[\pi\left(\alpha_{i}^{2}+\alpha_{s}^{2}\right)\right]^{1 / 2}}, \\
\mathcal{P}(\Omega, \theta)= & \frac{1}{\left[\pi \alpha_{s i}\left(\alpha_{i}^{2}+\alpha_{s}^{2}\left(1-n_{Z}^{2}\right)\right)\right]^{1 / 2}} \\
& \times \exp \left[-\left(\frac{v_{Z}-u_{Z}+\alpha_{s i} n_{Z} \boldsymbol{u} \cdot \boldsymbol{n}}{\left[\alpha_{s i}\left(\alpha_{i}^{2}+\alpha_{s}^{2}\left(1-n_{Z}^{2}\right)\right)\right]^{1 / 2}}\right)^{2}\right],
\end{aligned}
$$

where $n_{Z}$ is the LOS component of $\boldsymbol{n}$ and $\alpha_{s i}=\frac{\alpha_{s}^{2}}{\alpha_{i}^{2}+\alpha_{s}^{2}}$.

One may note that the main differences between the two components (collisional and radiative) are:

* The radiative component is affected by the outflow speed of the scattering ions through the Doppler dimming effect. The collisional component is not. 


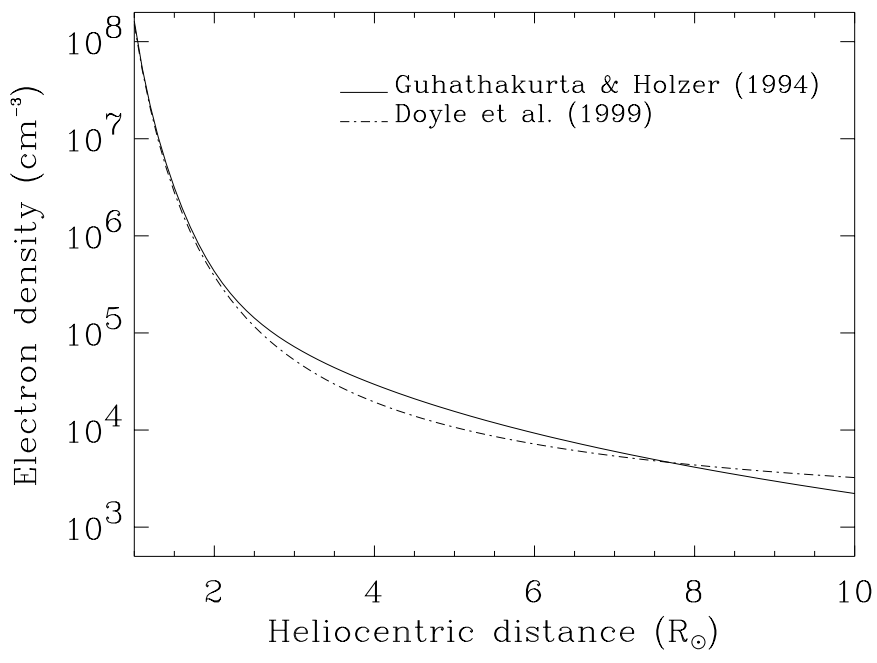

Fig. 3. Electron density given as a function of distance to the center of the Sun. Solid line: Guhathakurta \& Holzer (1994, polynomial fit); dot-dashed line: Doyle et al. (1999a,b, exponential fit).

* The two components do not shift in the same way. The component excited by electron collisions is more Doppler shifted than the radiatively excited one. For a given point on the line of sight, the Doppler shift of the collisional component reflects the real line-of-sight speed of the scattering atoms or ions. The shift of the radiative component, however, corresponds to only a fraction of this speed. This point is important for line profile broadening.

At low altitudes the radiative and collisional line components of the O VI doublet are similar in their sensitivity to the LOS speed. However, at high altitudes the Doppler shift of the collisional component increases (in absolute value) when moving away from the central area along the line of sight, but that of the radiative component increases at the beginning to saturate at a given limit.

\section{Choice of atmospheric parameters and Line-of-sight integration}

In order to integrate reliably along the line of sight it is necessary to model all the parameters that influence the scattering and emission processes. This includes the distribution of the scattering ions along the line of sight, the electron distribution, the macroscopic and microscopic velocity vector distributions, and in the case of polarized radiation the magnetic field distribution. Note that the magnetic field structure has an important indirect influence also on the intensity profile by the fact that it channels the solar wind and thus influences the velocity structure.

\subsection{Electron densities}

In Fig. 3 we plot the electron density stratifications for the polar coronal holes given by Guhathakurta \& Holzer (1994) (solid line) and Doyle et al. (1999a,b: Eq. (1) in both papers) (dotdashed line). The first is obtained from SKYLAB data and the second is from SoHO observations (using the SUMER, UVCS and LASCO instruments). Doyle et al. (1999a,b) applied an exponential fit to the measured densities as a function of the heliocentric distance:

$$
N_{\mathrm{e}}(\theta)=N_{\mathrm{e}_{\odot}} \exp \left[\frac{\mu m_{\mathrm{p}} G_{\odot} R_{\odot}^{2}}{k_{B} T_{\mathrm{av}}}\left(\frac{1}{r(\theta)}-\frac{1}{R_{\odot}}\right)\right],
$$

where $\mu$ is the mean atomic weight $(0.62) ; m_{\mathrm{p}}$ is the proton mass; $G_{\odot}$ is the solar surface gravitational acceleration $\left(274 \mathrm{~m} \mathrm{~s}^{-2}\right), R_{\odot}$ is the solar radius $\left(6.96 \times 10^{8} \mathrm{~m}\right)$ and $k_{\mathrm{B}}$ is the Boltzmann constant. $N_{\mathrm{e}_{\odot}}\left(=1.5 \times 10^{8} \mathrm{~cm}^{-3}\right)$ is the electron density at the base of the corona (often termed the solar surface in the solar wind literature) and $T_{\mathrm{av}}\left(=1.2 \times 10^{6} \mathrm{~K}\right)$ is the average of the electron and proton temperatures.

We note that the chosen density profile by Doyle et al. fits well the observations made below $8 R_{\odot}$. The density stratification beyond this distance does not affect our results since our range of integration along the line of sight is smaller than $8 R_{\odot}$. Hence, for the purpose of the present study it is not relevant how well the Doyle et al. densities fit the observations at greater distances.

The importance of the density profile resides therein that it influences the line profile integrated along the LOS directly, but also indirectly through the solar wind speed, which is determined via mass-flux conservation and thus depends on both the density stratification and the magnetic field. See the discussion in the following sections.

\subsection{Distribution of scattering ions along the line of sight}

We adopt the same profile for the distribution of scattering ions along the line of sight as the electron density but normalized to unity at the polar axis. This would be incorrect if we were calculating the absolute line intensities, but for determining the line profile only the relative variation is important.

\subsection{Magnetic field}

In the corona, plasma $\beta \ll 1$, so that the solar wind flows in directions specified by the field lines. In order to determine the solar wind velocity field vector $\boldsymbol{u}$ one needs to know the magnetic field vector at all locations along the LOS. For a description of the large scale coronal magnetic field we employ the analytical model of Banaszkiewicz et al. (1998) (Fig. 4), which is valid for the coronal conditions at the minimum of solar activity. This model combines dipole, quadrupole and equatorial current sheet contributions to the global field and is cylindrically symmetric with no azimuthal magnetic component. Smaller scale magnetic structure is neglected. These simplifications are reasonable for the range of heights that we are interested in, between 1.5 and $3.5 R_{\odot}$. For the free parameters of the model $M, Q, K$ and $a_{1}$ we use the values chosen by the authors: $M=1.789, Q=1.5, K=1.0$ and $a_{1}=1.538$ (see Banaszkiewicz et al. 1998).

In spite of its simplicity this model has been successful in reproducing the brightness distribution of the solar corona seen by the LASCO/SoHO coronagraphs (Brueckner et al. 1995) at solar activity minimum (Schwenn et al. 1997). Note in Fig. 4 that the open flux spreads superradially, leading to large 


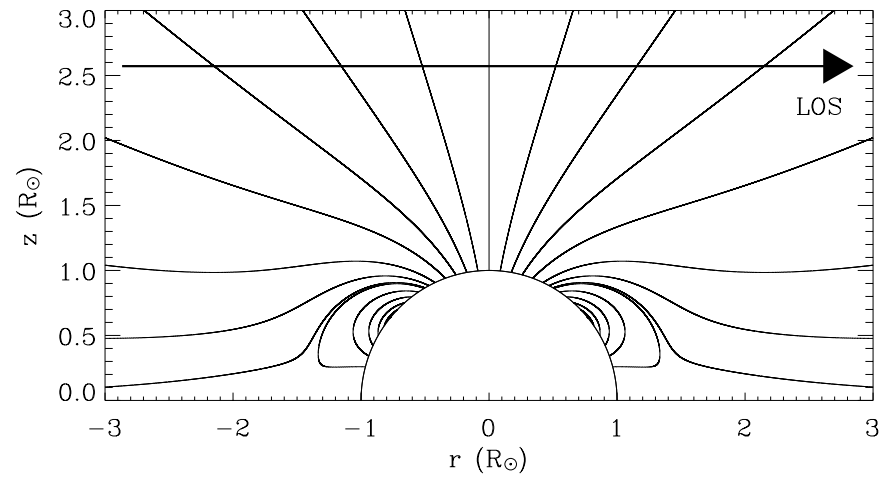

Fig. 4. Superradially expanding open magnetic field lines of the corona for the solar activity minimum according to the model by Banaszkiewicz et al. (1998). A sample LOS is plotted through the northern polar coronal hole (horizontal arrow).

LOS components of magnetic field and solar wind velocity, in particular at large $|Z|$ values.

\subsection{Solar wind velocity field}

The absolute outflow speed of the solar wind is prescribed at the base of the corona and is obtained at all locations of open flux from the mass and magnetic flux conservation equations (assuming that the flow follows the field lines):

$$
V(r)=\frac{N e\left(R_{\odot}, \theta\right)}{N e(r, \theta)} \frac{B(r, \theta)}{B\left(R_{\odot}, \theta\right)} V\left(R_{\odot}, \theta\right),
$$

where $N e\left(R_{\odot}, \theta\right), V\left(R_{\odot}, \theta\right)$ and $B\left(R_{\odot}, \theta\right)$ are the electron density, the outflow speed of the ions and the coronal magnetic field at the base of the solar corona (solar surface), respectively. $N e(r, \theta), V(r, \theta)$ and $B(r, \theta)$ are the same quantities at coordinates $(r, \theta)$. For $V\left(R_{\odot}, \theta\right)$ and $N e\left(R_{\odot}, \theta\right)$ we prescribe a constant, i.e. $\theta$-independent value within the coronal hole. At large $r$ this leads to a somewhat $(<10 \%)$ larger outflow speed at the edge of the coronal hole than over the pole due to the unequal expansion of the field lines. Since Ulysses measurements do not support such a solar wind speed gradient, we have also constructed a $V\left(R_{\odot}\right)$ that results in a $V\left(r=6 R_{\odot}\right)$ that is latitude independent. This $V\left(R_{\odot}\right)$ profile varies by less than $10 \%$ between the pole and the edge of the coronal hole. Adopting it does not affect our conclusions.

From the previous equations, we obtain the components of the outflow speed of the scattering ions by the following formulae

$$
V_{l}(\theta)=\frac{B_{l}(\theta)}{B(\theta)} V(\theta), \quad l=x, y, z,
$$

from which $B_{l}(\theta)$ and $B(\theta)$ can be computed by using the formulae given by Banaszkiewicz et al. (1998). To obtain $B_{\odot}$ corresponding to a given point on the LOS we track the magnetic field back to the solar surface from that point on the LOS.

Figure 5 displays the variation of the outflow speeds (top panels) and line-of-sight speeds (bottom panels) of the scattering ions for different projected heights above the solar limb. The speeds in the left-hand panels are obtained by using the density profile given by Guhathakurta \& Holzer (1994), while those on the right are deduced from the density profile given by Doyle et al. (1999a,b). Although the two density profiles are very similar (Fig. 3), they give significantly different outflow and line-of-sight speeds for the solar wind. For high altitudes, the outflow speed of the solar wind given by the density distribution by Guhathakurta \& Holzer (1994) decreases slightly when moving away from the the point of closest approach to the solar surface on the line of sight and then increases again. In general the solar wind speed given by the density profile of Doyle et al. increases much more rapidly with heliocentric distance so that it reaches larger values at heliocentric distances at which significant emission in O VI takes place. Note that the variation from pole to equator of the outflow speed over a circle with radius $6 R_{\odot}$ centered on Sun center is less than $10 \%$.

\subsection{Numerical integration along the LOS}

The frequency-dependent intensity of a given spectral line reaching the observer is obtained through Eq. (3). For the integration boundaries $Z_{0}$ we employ $Z_{0}=6 R_{\odot}$, since the line profile is found to acquire a significant contribution that affects its width out to this large $Z_{0}$ value.

The integral in Eq. (3) is over a spatial coordinate, but it is easier to integrate this equation over the scattering angle since all the terms are easily expressed in $\theta$. We use a subroutine that calculates points $\left(\theta_{1}, \theta_{2}, \theta_{3}, \ldots\right)$ on the LOS and the weights $\left(W_{\theta_{1}}, W_{\theta_{2}}, W_{\theta_{3}}, \ldots\right)$ associated with each. These weights are multiplied to the intensity profiles to compensate for the fact that the $\Delta Z_{i}=Z\left(\theta_{i+1}\right)-Z\left(\theta_{i}\right)$ are not equal. Since the integration is over a distance and the routine gives angles, one has to reevaluate the weights. We obtain

$$
W_{Z_{i}} \approx \frac{r_{0}}{\sin ^{2} \theta} W_{\theta_{i}} \sim \Delta Z_{i}
$$

\subsection{Turbulence velocity distribution}

The remaining parameter that needs to be prescribed is the turbulence velocity distribution. UVCS observations have previously suggested that the width of this distribution is extremely anisotropic. In order to maintain simplicity while emphasizing the effect of the density stratification we have chosen to prescribe an isotropic turbulence velocity distribution. Since we also want to compare our calculations with the observations of UVCS/SoHO we allow the Doppler width, $\alpha_{s}$, to increase gently with heliocentric distance (dot-dashed line in top panel of Fig. 7). The variation of $\alpha_{s}$ at each location along each line of sight is consistent with a purely radial variation of $\alpha_{s}$. The details of this assumption are unimportant, since we get almost identical results if instead we allow $\alpha_{s}$ to change with $r_{0}$ and keep it constant along each LOS. We do not attempt to obtain a best fit of the data, but did try different $\alpha_{s}$ profiles. We expect that the chosen $\alpha_{s}$ profile gives O VI doublet lines for which the widths and ratios are in the vicinity of as near as possible to being an optimal match for both density stratifications. 


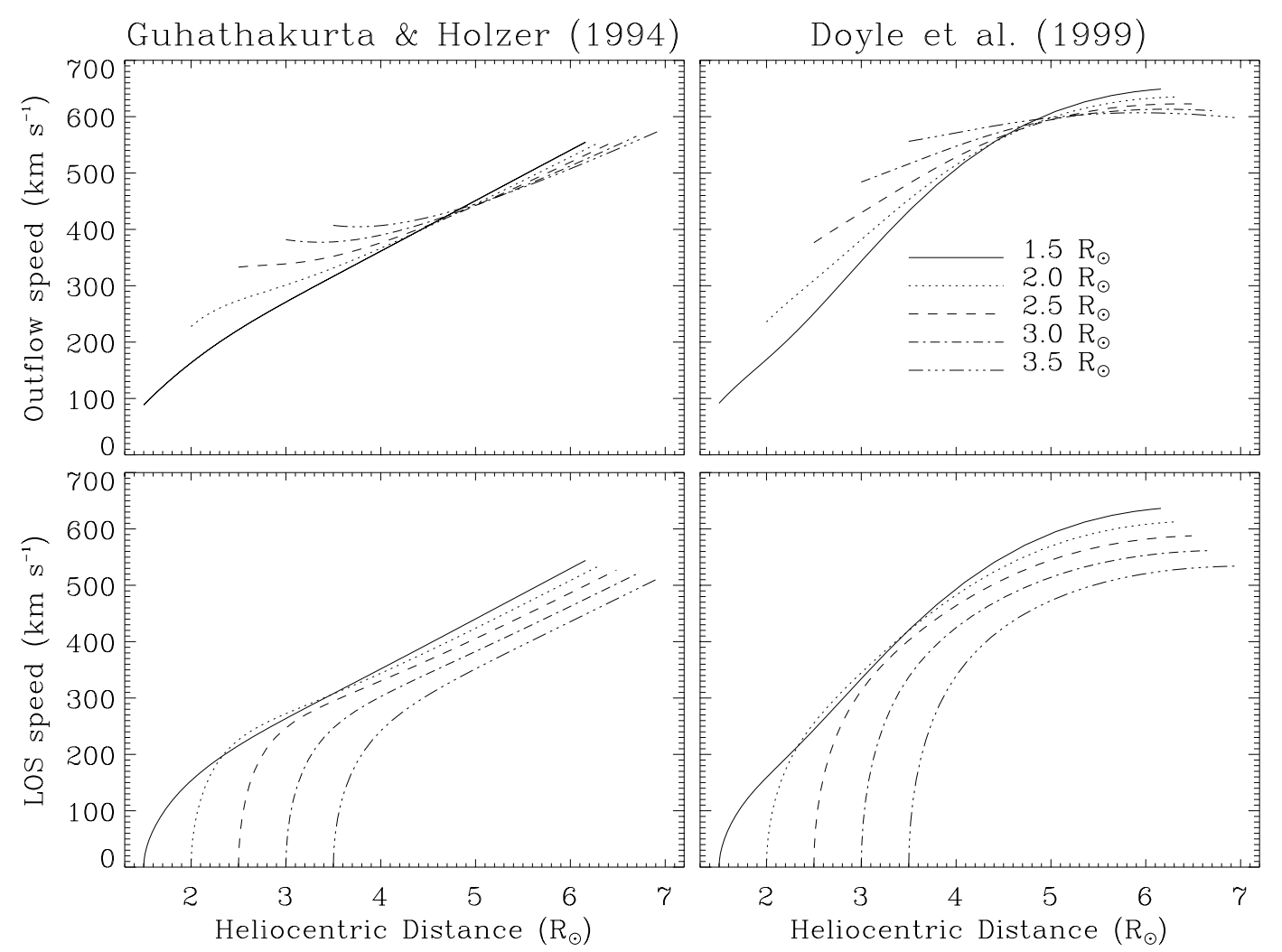

Fig. 5. Variation of the outflow speed of the solar wind (top panels) along the line of sight for different projected heliocentric distances and for the two density models (indicated above the panels). The minimum distance to Sun center reached by a particular ray is marked in the upper right panel. The bottom panels display the corresponding line-of-sight speeds.

\section{Results}

Using the parameters described in Sect. 4 we calculated the O vi doublet lines following Sect. 3. In the following we describe separately results obtained for two different latitudinal distributions of the outflow velocity at the solar surface.

\subsection{Constant $V\left(R_{\odot}\right)$}

Here we consider a constant outflow speed of the coronal plasma at the solar surface $\left(V\left(R_{\odot}\right)=9 \mathrm{~km} \mathrm{~s}^{-1}\right)$. This gives an outflow speed that is consistent within roughly $10 \%$ at $6 R_{\odot}$ (see Fig. 5). Using this boundary condition, two sets of computations were carried out, one for each considered density stratification. The resulting profiles of O VI $\lambda 1031.92$ are plotted for different projected heliocentric distances $r_{0}$ in Fig. 6. In the upper panel the profiles obtained with the Guhathakurta \& Holzer (1994) density are displayed. To keep differences in the line width from being hidden by the large changes in line strength all profiles have been normalized to the same amplitude. Clearly the width of the profiles increases strongly from 1.5 to $2.5 R_{\odot}$ projected heliocentric distance, but much less for larger distances. This is in contrast to the profiles plotted in the lower panel, resulting from the Doyle et al. densities. The widths of these continue to increase rapidly with increasing $r_{0}$. The shapes of all profiles are roughly Gaussian, although at large $r_{0}$ the profiles resulting from the Doyle et al. densities have a tendency to be more box-shaped. Nonetheless, all profiles were successfully fitted by Gaussian functions.

The e-folding widths $\left(V_{1 / e}\right)$ of the O VI $\lambda 1031.92$ profiles calculated for both density distributions are plotted in the top panel of Fig. 7 versus the projected distance of the line of sight from Sun center, $r_{0}$. At small $r_{0}$, the widths given by the two density models are comparable and widths obtained from UVCS data are reproduced without difficulty. The differences increase rapidly, however, when moving away from the solar limb. At large $r_{0}$ values $\left(>2.5 R_{\odot}\right)$ the widths obtained by using the Doyle et al. densities are comparable to those observed by UVCS, while those resulting from the Guhathakurta $\&$ Holzer (1994) densities are smaller by up to a factor of $\sim 1.5$ at $3.5 R_{\odot}$. The values obtained using the densities of Doyle et al., although smaller than the observed ones between $2 R_{\odot}$ and $2.5 R_{\odot}$, lie just at the edge of the domain covered by the scatter of the observed data points and the associated errors (see Cranmer et al. 1999c).

The bottom panel of Fig. 7 displays the line-integrated intensity ratios of the O VI doublet for the two density models. This ratio exhibits a marked dependence on radial distance, being well over two close to the Sun, then dropping rapidly to reach a minimum near $2.5 R_{\odot}$, before increasing again. Of particular interest is the fact that at heliocentric distances of 2.5 and $2.7 R_{\odot}$ the intensity ratio drops to very nearly unity, in agreement with the UVCS observations (taking into account the accuracy of the measurements). The intensity ratio turns out to be far less sensitive to the exact density profile chosen. 


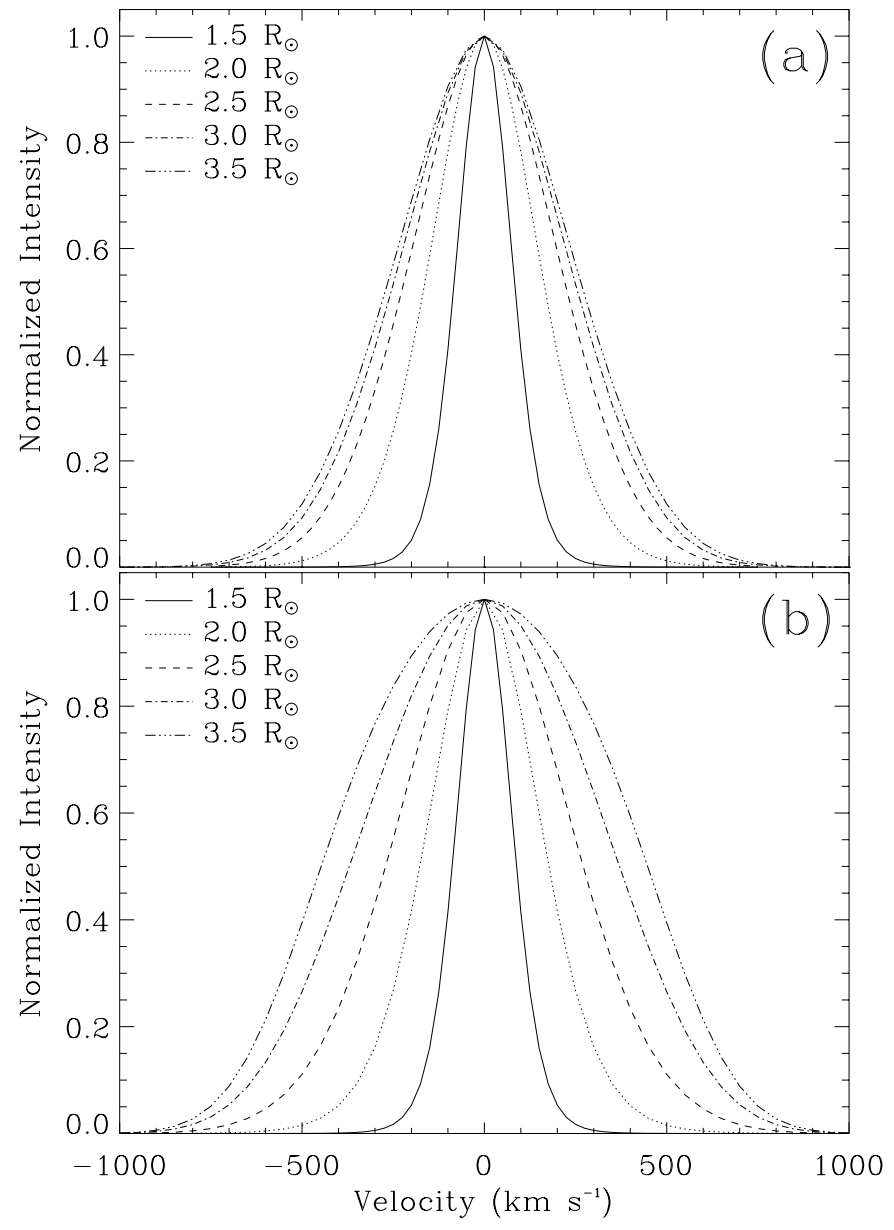

Fig. 6. Normalized profiles of the O VI $\lambda 1031.92$ line calculated for different heliocentric projected distances and different electron density profiles: a) Guhathakurta \& Holzer (1994) and b) Doyle et al. (1999a,b).

A comparison with Fig. 6 of Cranmer et al. (1999c) shows that the points computed using the Doyle et al. densities lie within the uncertainty of the measurements, with the possible exception of the two innermost points. Note that the influence of the integration over the LOS at these heights is not very important.

\subsection{Variable $V\left(R_{\odot}\right)$}

Observations recorded far away from the Sun by Ulysses show a nearly latitude-independent and constant speed of the fast solar wind. However, in the frame of the magnetic field model we consider here, the field strength at the solar surface drops by about a factor of approximately 1.5 from the pole to the edge of the polar hole. According to Eq. (14) and considering the fact that the magnetic field becomes nearly latitude-independent at a sufficient distance from the Sun, the solar wind originating from the edge of the polar hole should at the solar surface be 1.5 times faster that that rising from the pole.

To be consistent with the Ulysses measurements we introduce an outflow speed proportional to the strength of the magnetic field at the solar surface. The results obtained from this new boundary condition are shown in Fig. 8. They are very

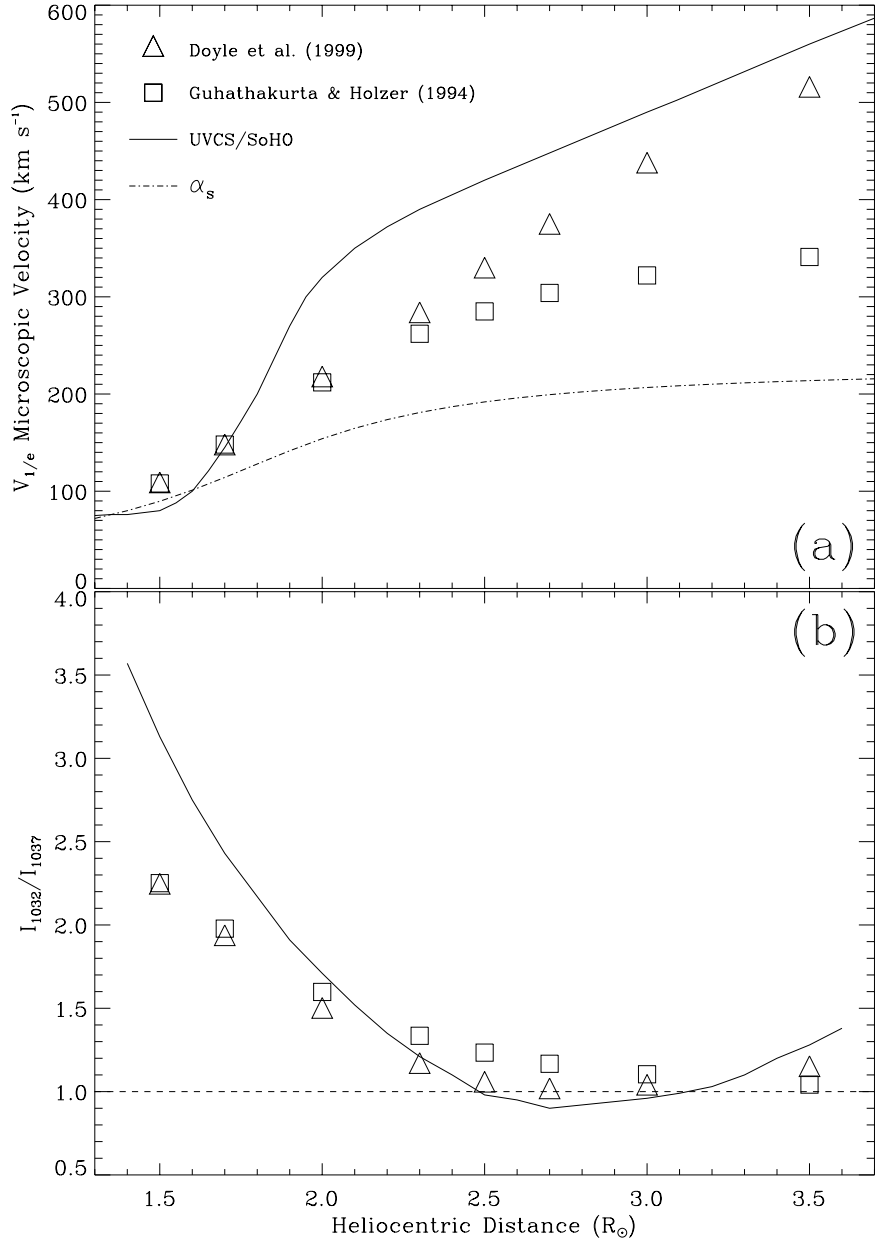

Fig. 7. a) $V_{1 / e}$, e-folding widths, of the LOS integrated profiles as a function of the projected heliocentric distance. Triangles: results of using the Doyle et al. density profile; squares: Guhathakurta \& Holzer (1994). The solid line represents the average of the UVCS data. Dotdashed line are the employed values of the Doppler width, $\alpha_{S}$, of the isotropic velocity distribution. b) Total intensity ratio of the $\mathrm{O}$ VI doublet.

similar to those obtained in the previous case of a constant outflow speed at the solar surface.

\section{Discussion}

We have considered the effect of the electron density distribution on the profiles of the O VI doublet emitted in the polar holes of the quiet solar corona using consistent models of the large scale magnetic field and solar wind structure, and taking into account the integration along the line of sight. The line widths and the total-intensity ratios of the $\mathrm{O}$ VI doublet obtained at different heights in the polar coronal holes are found to be strongly dependent on the electron density stratification. For densities given by Doyle et al. (1999a,b), the deduced widths and intensity ratios are comparable to those obtained through observations recorded by UVCS/SoHO, in spite of the fact that we only consider an isotropic kinetic temperature of the O vi ions. The density stratification given by Guhathakurta \& Holzer (1994), although similar, produces much lower widths of the LOS integrated profiles, in particular at large projected 


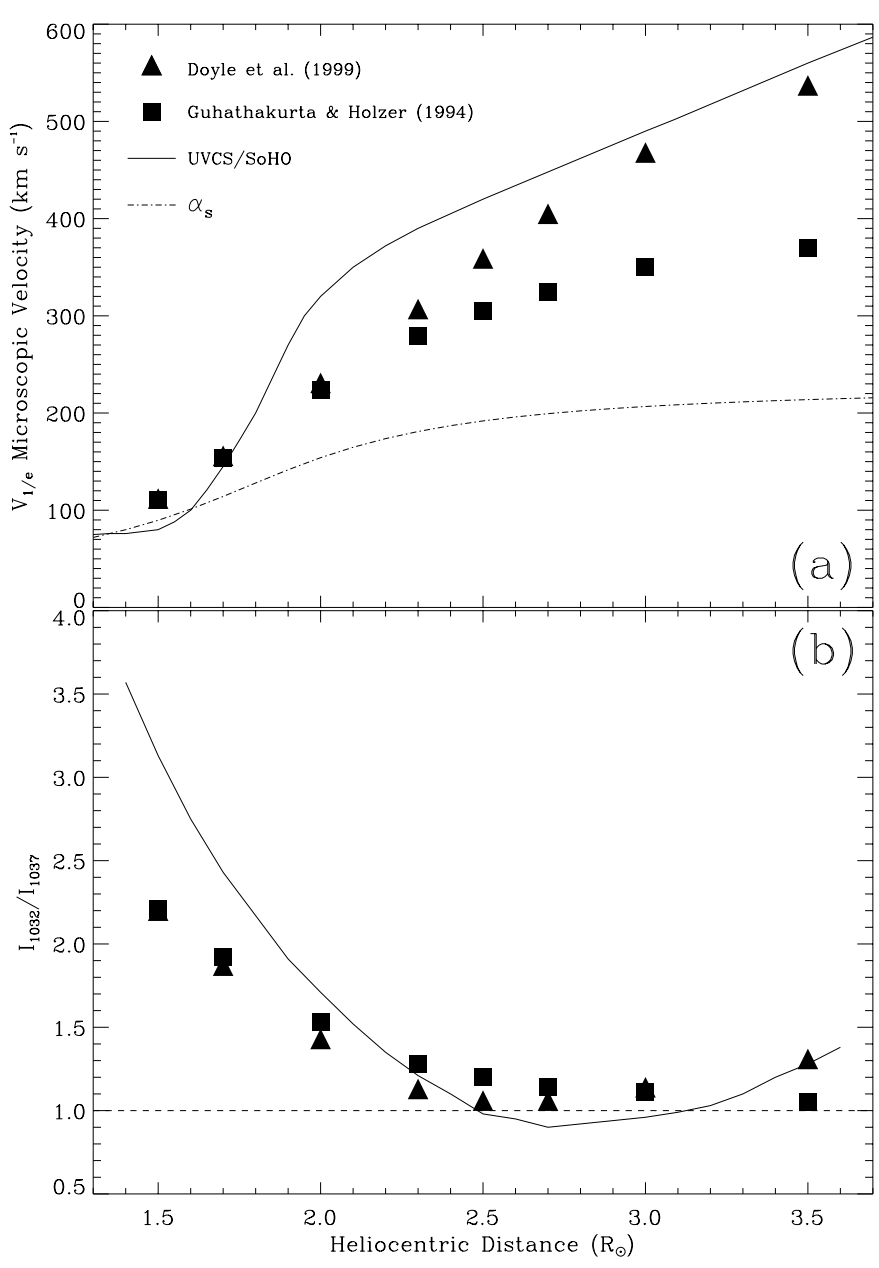

Fig. 8. Line widths (top panel) and total intensity ratios of the $\mathrm{O}$ VI doublet as a function of the projected heliocentric distance obtained by employing a $\theta$-dependent surface outflow velocity as boundary condition.

distances of the LOS to Sun center. Evidently, the width of the O VI doublet lines depends critically on the adopted density profile.

Our results demonstrate a clear need for further work, for example to test if the observed widths of the H I Lyman- $\alpha$ and $\mathrm{MgX}$ doublet lines are also roughly reproduced by the Doyle et al. densities for an isotropic velocity distribution. Such work should also include a study of whether more refined models for the coronal densities, but possibly also those of the magnetic field and solar wind, lead to an even better correspondence between observed profiles and those calculated assuming an isotropic velocity distribution. Also needed is a reappraisal of the importance of the anisotropy of the kinetic temperature of the heavy ions in the solar corona. This in turn has implications for the mechanisms of heating and acceleration of different species in the polar coronal holes.

Acknowledgements. The authors would like to thank Bernhard Fleck, Steven Cranmer, Bernd Inhester, Eckart Marsch and Klaus Wilhelm for helpful discussions and critical comments that greatly improved the paper.

\section{References}

Banaszkiewicz, M., Axford, W. I., \& McKenzie, J. F. 1998, A\&A, 337,940

Brueckner, G. E., Howard, R. A., Koomen, M. J., et al. 1995, Sol. Phys., 162, 357

Cranmer, S. R., Field, G. B., \& Kohl, J. L. 1999a, ApJ, 518, 937

Cranmer, S. R., Field, G. B., \& Kohl, J. L. 1999b, Space Sci. Rev., 87, 149

Cranmer, S. R., Kohl, J. L., Noci, G., et al. 1999c, ApJ, 511, 481

Domingo, V., Fleck, B., \& Poland, A. I. 1995, Sol. Phys., 162, 1

Doyle, J. G., Keenan, F. P., Ryans, R. S. I., Aggarwal, K. M., \& Fludra, A. 1999a, Sol. Phys., 188, 73

Doyle, J. G., Teriaca, L., \& Banerjee, D. 1999b, A\&A, 349, 956

Erdélyi, R., Doyle, J. G., Perez, M. E., \& Wilhelm, K. 1998, A\&A, 337, 287

Gabriel, A. H., Bely-Dubau, F., \& Lemaire, P. 2003, ApJ, 589, 623

Guhathakurta, M., \& Holzer, T. E. 1994, ApJ, 426, 782

Kohl, J. L., Esser, R., Gardner, L. D., et al. 1995, Sol. Phys., 162, 313

Kohl, J. L., Noci, G., Antonucci, E., et al. 1997, Sol. Phys., 175, 613

Kohl, J. L., Esser, R., Cranmer, S. R., et al. 1999, ApJ, 510, L59

Landi Degl'Innocenti, E. 1984, Sol. Phys., 91, 1

Li, X., Habbal, S. R., Kohl, J. L., \& Noci, G. 1998, ApJ, 501, L133

Noci, G., Kohl, J. L., \& Withbroe, G. L. 1987, ApJ, 315, 706

Raouafi, N.-E. 2000, Thèse de Doctorat, Université Paris XI, Orsay, France

Raouafi, N.-E. 2002, A\&A, 386, 721

Raouafi, N.-E., Sahal-Bréchot, S., Lemaire, P., \& Bommier, V. 2002, A\&A, 390, 691

Sahal-Bréchot, S., Bommier, V., \& Feautrier, N. 1998, A\&A, 340, 579

Schwenn, R., Inhester, B., Plunkett, S. P., et al. 1997, Sol. Phys., 175, 667

Warren, H. P., Mariska, J. T., Wilhelm, K., \& Lemaire, P. 1997, ApJ, 484, L91

Warren, H. P., Mariska, J. T., \& Wilhelm, K. 1998, ApJS, 119, 105

Wilhelm, K., Lemaire, P., Dammasch, I. E., et al. 1998, A\&A, 334, 685 